Rev. Int. Contam. Ambie. 32 (4) 385-397, 2016

DOI: 10.20937/RICA.2016.32.04.02

\title{
NIVELES DE METALES PESADOS EN SEDIMENTOS DE LA CUENCA DEL RÍO PUYANGO, ECUADOR
}

\author{
Abrahan MORA*, Diana JUMBO-FLORES, Max GONZÁLEZ-MERIZALDE y \\ Santos Amable BERMEO-FLORES
}

Universidad Nacional de Loja. Avenida Pío Jaramillo Alvarado, La Argelia, Provincia de Loja, Ecuador, C.P. 110113

*Autor para correspondencia: abrahanmora@hotmail.com

(Recibido julio 2015; aceptado febrero 2016)

Palabras clave: minería, mercurio, Sudamérica, contaminación

\section{RESUMEN}

En este estudio se determinaron las concentraciones de $\mathrm{Al}, \mathrm{Co}, \mathrm{Cu}, \mathrm{Fe}, \mathrm{Hg}, \mathrm{Ni}, \mathrm{Mn}$, $\mathrm{Pb}$ y $\mathrm{Zn}$ en muestras de sedimentos de fondo del río Puyango y de algunos de sus tributarios con el objeto de conocer los factores de enriquecimiento de estos elementos en los sedimentos. Las muestras de sedimento fueron sometidas a digestión ácida utilizando el método EPA 3050B, para luego ser analizadas por espectrofotometría de absorción atómica. Las muestras para el análisis de $\mathrm{Hg}$ total fueron digeridas con una mezcla de $\mathrm{HNO}_{3}, \mathrm{HCl}$ y $\mathrm{KMNO}_{4}$ y determinadas con la técnica de arrastre de vapor frío. Las mayores concentraciones de metales pesados fueron encontradas en los sedimentos colectados en el sector localizado después de la confluencia entre los ríos Calera y Amarillo. Los altos niveles de metales pesados y las bajas concentraciones de $\mathrm{Al}$ en los sedimentos de este sector indican que estos últimos están compuestos principalmente de mineral polimetálico, el cual ha sido descargado por las plantas procesadoras de mineral después del proceso de extracción de oro. Los sedimentos de las secciones media y baja del río Puyango se encuentran severamente contaminados con los elementos $\mathrm{Hg}, \mathrm{Cu}, \mathrm{Pb}, \mathrm{Zn}$ y $\mathrm{Mn}$, así como moderadamente contaminados con $\mathrm{Co}$ y Fe. Las altas concentraciones de $\mathrm{Hg}, \mathrm{Cu}, \mathrm{Pb}, \mathrm{Zn}, \mathrm{Mn}$ y Fe en los sedimentos de estos ríos pueden tener un efecto adverso para la biota acuática, de acuerdo con los criterios establecidos en normas internacionales.

Key words: mining, mercury, South America, contamination

\begin{abstract}
In this study the concentrations of $\mathrm{Al}, \mathrm{Co}, \mathrm{Cu}, \mathrm{Fe}, \mathrm{Hg}, \mathrm{Mn}, \mathrm{Ni}, \mathrm{Pb}$ and $\mathrm{Zn}$ were determined in bottom sediment samples of the Puyango River and its tributaries with the goal to know the enrichment factors of these elements in the sediments. Bulk sediment samples were digested using the EPA method 3050B and then analyzed by atomic absorption spectrometry. The samples for $\mathrm{Hg}$ determinations were digested with a mixture of $\mathrm{HNO}_{3}, \mathrm{HCl}$ y $\mathrm{KMNO}_{4}$ and then analyzed by the cold-vapor generation technique. The highest concentrations of heavy metals in sediments were found in the sector located after the Calera-Amarillo rivers confluence. The high levels of heavy metals and the low concentrations of $\mathrm{Al}$ in the bottom sediments of this sector indicate
\end{abstract}


that these riverine sediments are mainly composed by polymetallic mineral, which has been discharged from the ore processing plants after the gold extraction process. Sediments from the middle and lower Puyango River showed a severe contamination for $\mathrm{Hg}, \mathrm{Cu}, \mathrm{Pb}, \mathrm{Zn}$ and $\mathrm{Mn}$, and a moderate contamination for $\mathrm{Co}$ and $\mathrm{Fe}$. The high concentrations of $\mathrm{Hg}, \mathrm{Cu}, \mathrm{Pb}, \mathrm{Zn}, \mathrm{Mn}$ and $\mathrm{Fe}$ in the sediments of these rivers can have adverse effects on aquatic biota, according to international guidelines.

\section{INTRODUCCIÓN}

La cuenca del río Puyango abarca una superficie de unos $4400 \mathrm{~km}^{2}$ y se encuentra localizada al suroeste de Ecuador, cerca de la zona fronteriza entre el norte de Perú y el sur de Ecuador. En la zona alta de la cuenca se encuentra la región de PortoveloZaruma, en la cual se han llevado a cabo actividades mineras para la extracción de oro y plata desde el periodo precolonial. En esta zona existen más de 110 plantas procesadoras de mineral proveniente de unas 400 minas (plantas de beneficio), las cuales se encuentran localizadas a orillas de los principales tributarios del río Puyango (Guimaraes et al. 2011). Para la extracción de oro del mineral, las plantas de beneficio utilizan un proceso combinado de amalgamación con $\mathrm{Hg}$ y lixiviación con cianuro, lo que permite una mayor recuperación y beneficio económico. El proceso de molienda del mineral sulfuroso rico en metales pesados y su posterior lavado produce lixiviados mineros con un alto contenido de metales potencialmente tóxicos, los cuales se encuentran tanto en la fracción disuelta como en la fracción particulada. Debido al inadecuado manejo de estos lixiviados mineros, la mayoría de ellos llegan a los principales tributarios del río Puyango (ríos Calera y Amarillo), incorporando a ellos, grandes cantidades de cianuro, $\mathrm{Hg}$ y metales pesados (Tarras-Wahlberg 2002, Betancourt et al. 2005).

Hasta ahora, existen diversos trabajos que han analizado el contenido de algunos elementos como el $\mathrm{Zn}, \mathrm{Pb}, \mathrm{Cd}, \mathrm{Cu}$, As y $\mathrm{Hg}$ en agua, sedimentos suspendidos, sedimentos de fondo y biota en los ríos de la cuenca del Puyango (Tarras-Wahlberg et al. 2000, 2001). De manera similar, estudios más recientes han determinado la concentración de $\mathrm{Pb}, \mathrm{Mn}$, As y $\mathrm{Hg}$ en agua, sedimentos suspendidos y sedimentos de fondo de estos ríos, haciendo énfasis en las implicaciones que este tipo de contaminación tiene sobre las poblaciones humanas que viven aguas abajo de las zonas mineras (Betancourt et al. 2005, García et al. 2012). Aunque estos estudios exponen el alto contenido de los elementos mencionados en diferentes matrices ambientales, no hacen referencia al contenido de otros elementos como el Ni, Co y Fe, los cuales también pueden estar enriquecidos en los sedimentos. Además, tampoco determinan la magnitud del enriquecimiento de metales pesados en los sedimentos de los ríos de la cuenca. Por consiguiente, el objetivo de este trabajo fue analizar la concentración de ciertos elementos $(\mathrm{Al}, \mathrm{Co}, \mathrm{Cu}$, $\mathrm{Fe}, \mathrm{Hg}, \mathrm{Mn}, \mathrm{Ni}, \mathrm{Pb}$ y $\mathrm{Zn}$ ) en sedimentos de algunos sectores de la cuenca del río Puyango y determinar el factor de enriquecimiento que presentan los metales pesados en esos sedimentos.

\section{MATERIALES Y MÉTODOS}

\section{Zona de estudio}

El río Puyango posee un caudal promedio anual de unos $88 \mathrm{~m}^{3} / \mathrm{s}$ antes de alcanzar la quebrada Cazaderos, en la frontera Perú-Ecuador. De allí en adelante, el canal principal del río es territorio completamente peruano y se denomina río Tumbes. Hacia sus cabeceras, el río Puyango recibe a sus tres principales tributarios (ríos Calera, Amarillo y Pindo), los cuales poseen un caudal promedio anual de $9 \mathrm{~m}^{3} / \mathrm{s}, 15 \mathrm{~m}^{3} / \mathrm{s}$ y $24 \mathrm{~m}^{3} / \mathrm{s}$, respectivamente (Tarras-Wahlberg et al. 2001). Las plantas de procesamiento del mineral aurífero o plantas de beneficio se encuentran ubicadas en las poblaciones de Portovelo y Zaruma, principalmente hacia las orillas de los ríos Calera y Amarillo. No obstante, después de la confluencia entre los ríos Calera y Amarillo y antes de la confluencia con el río Pindo, existen algunas lagunas de lixiviados y plantas de beneficio (Fig. 1). Esta zona se encuentra ubicada en la Provincia de El Oro, en las colinas y laderas occidentales de la cordillera de los Andes, al sur de Ecuador. El régimen hidrológico del río Puyango y sus tributarios se encuentra determinado por una temporada seca y una temporada lluviosa. Durante la época seca, la cual se extiende desde finales de mayo hasta principios de enero, el río Puyango posee bajos valores de caudal (caudal mínimo promedio de $21 \mathrm{~m}^{3} / \mathrm{s}$ ), mientras que durante la época de lluvias (desde finales de enero hasta principios de mayo), el caudal del río aumenta considerablemente, alcanzando un valor máximo promedio de $230 \mathrm{~m}^{3} / \mathrm{s}$ (Tarras-Walhberg y Lane 2003). Asimismo, los ríos 


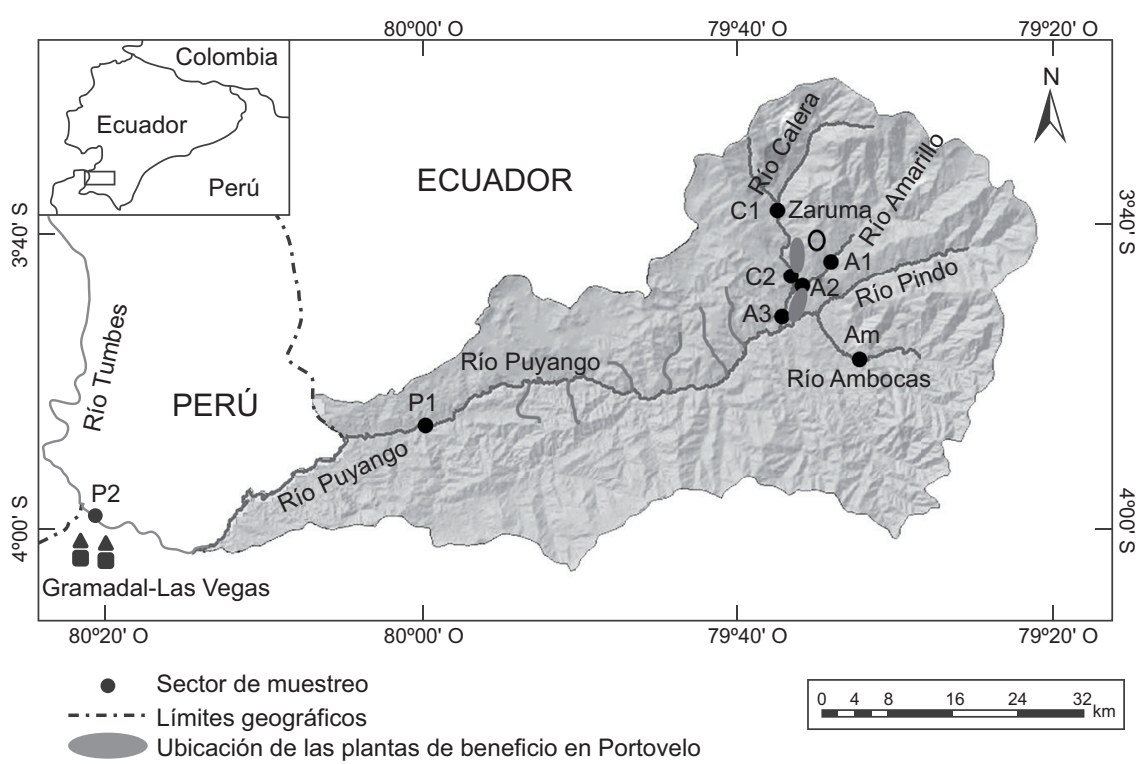

Fig. 1. Ubicación de los sectores de muestreo de sedimentos en la cuenca del río Puyango, Ecuador

Calera, Amarillo y Pindo presentan un régimen hidrológico determinado por la estacionalidad de las precipitaciones, con valores mínimos promedios de $2.1 \mathrm{~m}^{3} / \mathrm{s}$, $3.5 \mathrm{~m}^{3} / \mathrm{s}$ y $6.4 \mathrm{~m}^{3} / \mathrm{s}$, respectivamente, durante la sequía y valores máximos promedios de $20 \mathrm{~m}^{3} / \mathrm{s}, 33 \mathrm{~m} / \mathrm{s} \mathrm{y}$ $56 \mathrm{~m}^{3} / \mathrm{s}$, respectivamente, durante el periodo lluvioso (Tarras-Walhberg y Lane 2003). Debido a las variaciones de altitud y topografía, el clima a lo largo de la cuenca del Puyango es muy variado. En la zona de Zaruma-Portovelo, la vegetación es bosque húmedo templado y la temperatura oscila entre los 14 y $\operatorname{los} 22^{\circ} \mathrm{C}$, con una precipitación media anual de $1500 \mathrm{~mm}$ (Gobierno Provincial Autónomo de El Oro 2014). No obstante, los valores de temperatura aumentan y las precipitaciones disminuyen hacia las zonas bajas de la cuenca. En la parte baja, se encuentran los bosques secos de la provincia de Loja, zona que presenta un clima tropical semiárido, con una temperatura y una precipitación media anual de $23{ }^{\circ} \mathrm{C}$ y $500 \mathrm{~mm}$, respectivamente.

\section{Colecta de muestras de sedimento}

Se colectaron muestras de sedimento en 8 sectores distribuidos a lo largo de la cuenca de río Puyango. En el río Calera se tomaron muestras de sedimento en 2 sectores (antes y después de las plantas de beneficio), mientras que el río Amarillo fue muestreado en 3 diferentes sectores (uno localizado antes de las plantas de beneficio, otro después de las plantas de beneficio y el último después de la confluencia entre los ríos Calera y Amarillo). El río Ambocas, uno de los principales tributarios del río Pindo, fue muestreado en un solo sector, mientras que en el río Puyango se tomaron muestras hacia las cercanías de la población de Puyango (cuenca media) y en la zona baja de la cuenca, localizada en los bosques secos de la provincia de Loja (sector Gramadal-Las Vegas). En cada sector, se colectaron tres muestras de sedimento mediante una pala de polietileno previamente lavada con agua desionizada. El muestreo se realizó durante la finalización del periodo seco (enero 2015), aprovechando el bajo nivel que presentan los ríos durante esta temporada. Durante esta época del año, el material polimetálico molido aportado por las plantas de beneficio, el cual es rico en metales pesados, tiende a sedimentar debido a la baja velocidad de las aguas. Sin embargo, durante la época lluviosa este material se re-suspende y es arrastrado hacia la sección más baja de la cuenca como resultado del incremento en la corriente de las aguas (Betancourt et al. 2005), haciendo que disminuyan las concentraciones de metales pesados en el sedimento de fondo (Tarras-Wahlberg et al. 2001). Por consiguiente, el análisis de las muestras de sedimento colectadas durante la época seca proporciona una mayor información del impacto producido por la minería sobre este sistema hídrico. Las muestras de sedimento se almacenaron individualmente en bolsas de plástico y se colocaron en hieleras para su posterior traslado. El cuadro I muestra la codificación y las coordenadas geográficas de los sectores de muestreo, mientras que la figura 1 muestra la ubicación de cada uno de estos sectores en la cuenca del río Puyango. 
CUADRO I. CODIFICACIÓN, ALTITUD Y LOCALIZACIÓN DE LOS SECTORES DE MUESTREO DE SEDIMENTOS EN LOS RÍOS AMBOCAS, CALERA, AMARILLO Y PUYANGO

\begin{tabular}{|c|c|c|c|c|}
\hline Río & $\begin{array}{l}\text { Identificador } \\
\text { del Sector }\end{array}$ & $\begin{array}{l}\text { Altitud } \\
\text { (msnm) }\end{array}$ & Latitud & Longitud \\
\hline Ambocas & $\mathrm{Am}$ & 649 & $3^{\circ} 47^{\prime} 10.1 " \mathrm{~S}$ & $79^{\circ} 35$ \\
\hline Amarillo & A1 & 669 & $3^{\circ} 42^{\prime} 40.6 ” S$ & $79^{\circ} 36^{\prime} 21.8^{\prime \prime} \mathrm{O}$ \\
\hline Amarillo & A2 & 608 & $3^{\circ} 43^{\prime} 41.4$ '’S & 79³8'02.6”O \\
\hline Amarillo & A3 & 548 & $3^{\circ} 46^{\prime} 02.9{ }^{\prime \prime S}$ & 79³8'49.7'O \\
\hline Calera & $\mathrm{C} 1$ & 805 & $3^{\circ} 38^{\prime} 50.2 " \mathrm{~S}$ & $79^{\circ} 39^{\prime} 00.6 " \mathrm{O}$ \\
\hline Calera & $\mathrm{C} 2$ & 611 & $3^{\circ} 43^{\prime} 20.8 ” S$ & $79^{\circ} 38^{\prime} 01.0$ 'O \\
\hline Puyango & $\mathrm{P} 1$ & 299 & $3^{\circ} 52^{\prime} 29.7^{\prime \prime S}$ & $80^{\circ} 02^{\prime} 58.0$ 'O \\
\hline Puyango & P2 & 154 & 3०59'07.2”S & $80^{\circ} 21^{\prime} 37.1^{\prime \prime O}$ \\
\hline
\end{tabular}

\section{Tratamiento y análisis de muestras}

En el laboratorio, las muestras de sedimentos fueron colocadas en platos de polietileno y secadas a $40^{\circ} \mathrm{C}$ en una estufa. Posteriormente, estas muestras se disgregaron con un mortero de porcelana y se tomó una nueva muestra representativa por el método del cuarteo. Los metales pesados que son lixiviados por actividades industriales y mineras suelen acumularse tanto en los limos como en las arcillas (tamaño de grano $<63 \mu \mathrm{m}$ ) que se encuentran presentes en los sedimentos de los sistemas ribereños y marinocosteros, por lo cual es conveniente realizar un tamizado de la muestra de sedimento para la posterior determinación de metales en esta fracción fina. Sin embargo, debido a que el sedimento del río Puyango se encuentra constituido principalmente por el mineral polimetálico que es procesado en la zona alta de la cuenca (Betancourt et al. 2005), los metales pesados pueden encontrarse tanto en la fracción fina como en la fracción gruesa, motivo por el cual las muestras colectadas no fueron previamente tamizadas.

Se empleó el método EPA 3050B $\left(\mathrm{HNO}_{3}+\mathrm{HCl}\right.$ $+\mathrm{H}_{2} \mathrm{O}_{2}$; EPA 1996) para la digestión y posterior determinación de los elementos ambientalmente disponibles en los sedimentos colectados. Este método consiste en una fuerte digestión ácida que produce una disolución selectiva de elementos intercambiables, oxihidróxidos de Fe-Mn, carbonatos y elementos enlazados tanto a la materia orgánica como a estructuras minerales (Kaasalainen y Yli-Halla 2003). Este procedimiento consistió en tomar $1 \mathrm{~g}$ de cada muestra de sedimento y someterlo a digestión con reflujo usando $10 \mathrm{~mL}$ de solución de $\mathrm{HNO}_{3}$ (1:1) a $95^{\circ} \mathrm{C}$ por $10 \mathrm{~min}$, evitando la ebullición para impedir la pérdida de ciertos elementos como el $\mathrm{Pb}$. Posteriormente, se agregaron $5 \mathrm{~mL}$ de $\mathrm{HNO}_{3}$ concentrado $(65 \%)$ a cada muestra y se calentó nuevamente por
30 min con reflujo. A continuación, se evaporaron las muestras sin ebullición por $2 \mathrm{~h}$ hasta obtener $5 \mathrm{~mL}$ de solución. Después de enfriar y agregar $2 \mathrm{~mL}$ de agua desionizada y $3 \mathrm{~mL}$ de $\mathrm{H}_{2} \mathrm{O}_{2}(30 \%)$, las muestras fueron calentadas a $95{ }^{\circ} \mathrm{C}$ por $2 \mathrm{~h}$, lo que permitió la evaporación hasta obtener $5 \mathrm{~mL}$ de solución. Como último paso, se agregaron $10 \mathrm{~mL}$ de $\mathrm{HCl}$ concentrado a cada muestra y después se calentaron a $95^{\circ} \mathrm{C}$ por $15 \mathrm{~min}$. Una vez ambientadas, las muestras fueron filtradas a través de membranas Whatman 41 para evitar la presencia de pequeñas partículas que puedieran tapar el nebulizador del equipo de absorción atómica durante los análisis. Los filtrados fueron aforados a $100 \mathrm{~mL}$ y colocados en envases de polietileno previamente lavados con ácido nítrico y agua desionizada. Dichos extractos fueron almacenados a $4{ }^{\circ} \mathrm{C}$ para la posterior determinación de metales pesados. Este mismo procedimiento de digestión se utilizó para preparar los blancos y los patrones usados para realizar el control de calidad de los análisis. Las determinaciones de los metales en los extractos se realizaron por espectrofotometría de absorción atómica con un espectrofotómetro marca Shimatzu modelo AA-6800 y lámpara de deuterio para la corrección de la absorbancia no atómica. Para la preparación de los patrones utilizados en las curvas de calibración se emplearon estándares certificados de $1000 \mathrm{mg} / \mathrm{L}$ marca Accustandard. Los elementos $\mathrm{Fe}, \mathrm{Co}, \mathrm{Cu}, \mathrm{Mn}, \mathrm{Ni}, \mathrm{Pb}$ y $\mathrm{Zn}$ fueron analizados por la llama de aire-acetileno, mientras que el Al fue medido a través de la llama de óxido nitroso-acetileno, agregando $2000 \mathrm{mg} / \mathrm{L}$ de $\mathrm{K}$ tanto a extractos como a patrones, para así evitar la ionización del analito.

Para la determinación de $\mathrm{Hg}$ en las muestras de sedimento, se tomó $0.1 \mathrm{~g}$ de cada muestra y se sometió a digestión con $\mathrm{HNO}_{3}, \mathrm{HCl}$ y $\mathrm{K}_{2} \mathrm{MnO}_{4}$, de acuerdo con APHA (2012). El método consiste en la digestión del sedimento con esos tres reactivos a $95^{\circ} \mathrm{C}$ en un baño de agua. Una vez frías las muestras, se agregaron $2 \mathrm{~mL}$ de una solución de clorhidrato de hidroxilamina (12\%) a cada una, para eliminar el exceso de permanganato. Se determinó la concentración de $\mathrm{Hg}$ por absorción atómica a través del método de arrastre de vapor frío con un generador de hidruros marca Shimatzu modelo HVG-1.

La precisión de los análisis (valor basado en tres muestras colectadas en cada sector de muestreo) varió entre 1 y $20 \%$ para todos los elementos estudiados. El material de referencia de sedimentos de lago del Organismo Internacional de Energía Atómica (IAEA-SL1) se utilizó para determinar la exactitud del procedimiento analítico. El cuadro II muestra los límites de detección para los elementos 
CUADRO II. LÍMITES DE DETECCIÓN DE LOS ELEMENTOS DETERMINADOS Y COMPARACIÓN DE LOS RESULTADOS DE LOS ANÁLISIS REALIZADOS EN EL MATERIAL DE REFERENCIA DEL ORGANISMO INTERNACIONAL DE ENERGÍA ATÓMICA (IAEA-SL1, en inglés) CON LOS VALORES RECOMENDADOS

\begin{tabular}{lcccccc}
\hline Elemento & $\begin{array}{c}\text { Límite de } \\
\text { detección }\end{array}$ & $\begin{array}{c}\text { Valor } \\
\text { obtenido }\end{array}$ & $\begin{array}{c}\text { Valor } \\
\text { recomendado }\end{array}$ & $\begin{array}{c}\text { Diferencia } \\
\text { relativa (\%) }\end{array}$ & $\begin{array}{c}\text { Intervalo de } \\
\text { confianza 95 \% }\end{array}$ \\
\hline $\mathrm{Mn}(\mathrm{mg} / \mathrm{kg})$ & 3 & 3560 & \pm 54 & 3460 & 2.9 & $3300-3620$ \\
$\mathrm{Zn}(\mathrm{mg} / \mathrm{kg})$ & 1 & 193 & \pm 4 & 223 & 13.5 & $213-233$ \\
$\mathrm{Fe}(\mathrm{mg} / \mathrm{kg})$ & 8 & 62282 & \pm 160 & 67400 & 7.6 & $65700-69100$ \\
$\mathrm{~Pb}(\mathrm{mg} / \mathrm{kg})$ & 10 & $31.5 \pm 1.3$ & 37.7 & 16.4 & $30.3-45.1$ \\
$\mathrm{Cu}(\mathrm{mg} / \mathrm{kg})$ & 4 & $27.1 \pm 0.4$ & 30 & 9.7 & $24-36$ \\
$\mathrm{Ni}(\mathrm{mg} / \mathrm{kg})$ & 8 & 37 & \pm 2 & 44.9 & 17.6 & $36.9-53.9$ \\
$\mathrm{Co}(\mathrm{mg} / \mathrm{kg})$ & 3 & $17.5 \pm 0.3$ & 19.8 & 11.6 & $18.3-21.3$ \\
$\mathrm{Hg}(\mathrm{mg} / \mathrm{kg})$ & 0.015 & $0.08 \pm 0.04$ & 0.13 & 37.8 & $0.08-0.18$ \\
\hline
\end{tabular}

analizados por espectrofotometría de absorción atómica y compara los resultados de los análisis realizados en el material de referencia con los valores recomendados por el Organismo Internacional de Energía Atómica (OIEA). Aunque la diferencia relativa entre los resultados obtenidos y los valores recomendados por el OIEA no fue superior al $18 \%$ en la mayoría de los casos, el valor presentado para el análisis de $\mathrm{Hg}$ mostró una diferencia relativa de un $37.8 \%$. Sin embargo, este valor se encuentra dentro del intervalo de confianza del $95 \%$ estimado por el OIEA para las determinaciones de $\mathrm{Hg}$ en este material de referencia.

\section{Análisis estadísticos}

Se utilizó el programa Statistica 7.0 para generar los diagramas de dispersión utilizados para calcular los factores de enriquecimiento normalizados. Además, se utilizó la prueba t de Student para comparar los valores de concentración media de los elementos en las muestras de sedimentos. Las regresiones lineales y las comparaciones entre muestras fueron consideradas significativas para un valor de $\mathrm{p}<0.05$.

\section{RESULTADOS}

La figura 2 muestra las concentraciones promedio y las desviaciones estándar de los elementos determinados en los sedimentos de los ríos Calera, Amarillo, Ambocas y Puyango. Los sedimentos colectados en el río Ambocas presentaron las concentraciones más bajas de los elementos estudiados, lo que indica que este río no es afectado por las actividades mineras que se desarrollan en la cuenca. Las concentraciones de los elementos determinados en los sedimentos colectados en los ríos Amarillo y Calera en los sectores ubicados antes de las plantas de beneficio (sectores $\mathrm{C} 1$ y A1), presentaron valores superiores a los observados en los sedimentos del río Ambocas, posiblemente debido a la presencia de depósitos masivos de sulfuros polimetálicos, los cuales son abundantes en la parte alta de la cuenca del Puyango. Las concentraciones de los metales $\mathrm{Mn}, \mathrm{Zn}, \mathrm{Cu}, \mathrm{Pb}$ y $\mathrm{Hg}$ presentaron un incremento significativo $(\mathrm{p}<0.05)$ en los sedimentos colectados en los sectores $\mathrm{C} 2$ y A2 de los ríos Calera y Amarillo (después de las plantas de beneficio) en relación con los valores observados en los sectores $\mathrm{C} 1$ y A1. Los sedimentos del sector A3 presentaron las mayores concentraciones de $\mathrm{Zn}, \mathrm{Fe}, \mathrm{Cu}, \mathrm{Pb}$, Ni y Co encontradas en la cuenca durante este estudio. Hacia la sección media y baja de la cuenca, se observó una disminución significativa en la concentración de los metales $\mathrm{Zn}, \mathrm{Fe}, \mathrm{Cu}, \mathrm{Pb}$, Ni y Co en los sedimentos de fondo del Puyango, en comparación con las muestras colectadas en el sector A3. Sin embargo, no se observaron diferencias significativas en la concentración de algunos metales ( $\mathrm{Mn}, \mathrm{Zn}, \mathrm{Fe}, \mathrm{Cu}, \mathrm{Pb}$ y $\mathrm{Co}$ ) entre los sedimentos tomados en la sección media (sector P1) y en la sección baja (sector P2) del río Puyango.

En general, las concentraciones de los elementos estudiados en los sedimentos colectados decrecieron en el orden $\mathrm{Fe}>\mathrm{Al}>\mathrm{Mn}>\mathrm{Zn}>\mathrm{Cu}>\mathrm{Pb}>\mathrm{Co}>\mathrm{Ni}$ $>\mathrm{Hg}$ en los sectores no impactados o poco impactados (Am, C1 y A1). Sin embargo, en los sectores ya impactados por lixiviados mineros $(\mathrm{C} 2, \mathrm{~A} 2, \mathrm{~A} 3$, P1 y P2), el Zn desplaza al Mn como elemento más abundante en los sedimentos. Inclusive, en el sector $\mathrm{A} 3$, las concentraciones de $\mathrm{Zn}$ fueron superiores a las concentraciones de Al. En los sectores $\mathrm{C} 2$ y A3 también se observa que las concentraciones de $\mathrm{Pb}$ y $\mathrm{Cu}$ son muy altas en los sedimentos, siendo superiores a las concentraciones de $\mathrm{Mn}$. 

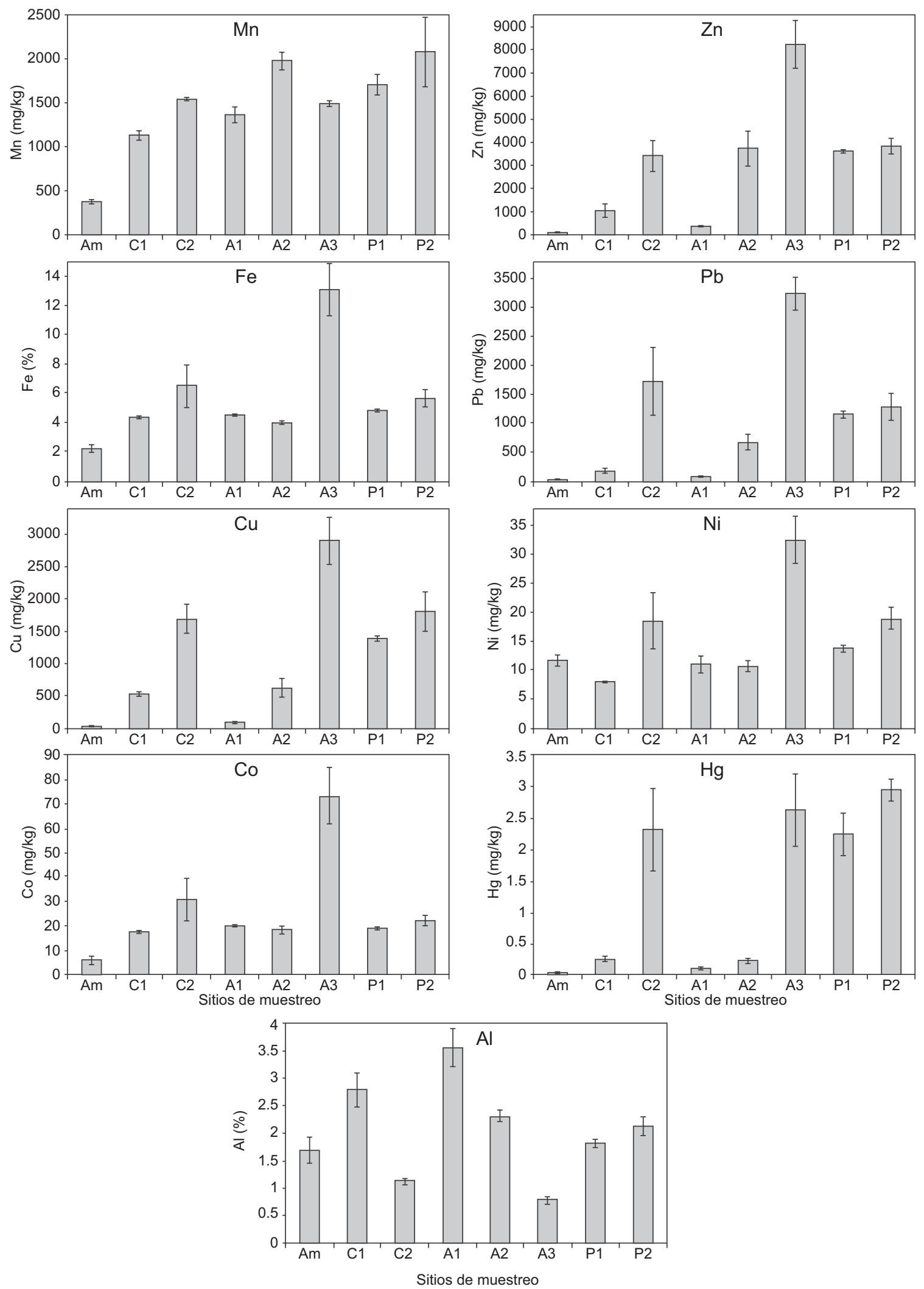

Fig. 2. Valores de concentración promedio y desviaciones estándar de los elementos determinados en los sedimentos de los ríos Ambocas, Calera, Amarillo y Puyango 


\section{DISCUSIÓN}

\section{Distribución espacial de los metales pesados en sedimentos de la cuenca del río Puyango}

Como se mencionó anteriormente, existe un incremento significativo de la concentración de $\mathrm{Mn}, \mathrm{Zn}, \mathrm{Pb}$, $\mathrm{Cu}$ y $\mathrm{Hg}$ en los sedimentos colectados en los sectores C2 y A2 en comparación con los valores observados en los sedimentos de los ríos Amarillo y Calera antes de las plantas de beneficio. Este incremento es producido por la incorporación de lixiviados mineros procedentes de las plantas procesadoras de mineral, los cuales son tratados inadecuadamente antes de ser vertidos a estos ríos. De manera similar, las altas concentraciones de $\mathrm{Zn}, \mathrm{Fe}, \mathrm{Cu}, \mathrm{Pb}$, Ni y Co encontradas en el sector A3 indican que existe un incremento en el flujo de efluentes procedentes de las lagunas de lixiviado y plantas de beneficio que se encuentran después de la confluencia entre los ríos Amarillo y Calera. Hacia la sección media de la cuenca (sector P1), la concentración de estos elementos disminuye considerablemente, probablemente debido a la incorporación de material particulado no contaminado aportado por el río Pindo y por los demás tributarios que discurren en esta sección. Sin embargo, la concentración de estos elementos permanece casi constante hacia la sección baja de la cuenca (sector P2), hecho que podría estar relacionado con una disminución en los valores de precipitación media anual en esta zona, que posee características propias de una región semiárida. Por consiguiente, al haber valores bajos de precipitación y escorrentía, el caudal y el flujo de material particulado no contaminado aportado por los tributarios de la sección baja de la cuenca es muy bajo, lo que induce a que existan pocas variaciones en la concentración de metales pesados en sedimentos a lo largo del canal principal del bajo río Puyango.

El cuadro III compara los resultados obtenidos en este estudio con los trabajos anteriores realizados en la cuenca del Puyango y con las normas para la protección y el manejo de sedimentos acuáticos del Ministerio del Ambiente de Ontario (Persaud et al. 1993). Las concentraciones de $\mathrm{Zn}, \mathrm{Pb}$ y Cu determinadas en este estudio en los sedimentos de fondo de los ríos Calera y Amarillo (sectores C2 y A2), fueron entre un 50 y $75 \%$ menores a los valores medidos entre 1998 y 1999 por Tarras-Wahlberg et al. (2001). Sin embargo, los valores de concentración de estos elementos en los sedimentos de la sección media de la cuenca (sector P1) fueron muy parecidos a los reportados por Tarras-Wahlberg et al. (2001) para este mismo sector. De igual forma, los valores de $\mathrm{Mn}$ y $\mathrm{Pb}$ presentados en este estudio estuvieron dentro de los rangos de concentración reportados por García et al. (2012) para los sedimentos de la cuenca del Puyango.

Respecto al $\mathrm{Hg}$, las concentraciones de este elemento en los sedimentos de los sectores C2, P1 y P2 fueron muy superiores a los valores reportados en estudios previos (Tarras-Wahlberg et al. 2001, García et al. 2012). En principio, esto podría ser consecuencia de un incremento en la actividad minera que se desarrolla en la región. Las cifras de producción de oro proveniente de la pequeña minería y de la minería artesanal en Ecuador han registrado un crecimiento sostenido en estos últimos años (ARCOM 2016). No obstante, estudios realizados por Mainville et al. (2006) han demostrado que la deforestación en las zonas andinas de Ecuador puede propiciar la lixiviación

CUADRO III. CONCENTRACIONES PROMEDIO DE LOS ELEMENTOS DETERMINADOS EN LOS SEDIMENTOS DE LA CUENCA DEL RÍO PUYANGO Y COMPARACIÓN CON LOS VALORES OBTENIDOS EN OTROS ESTUDIOS Y CON LOS VALORES DEL NIVEL DE BAJO EFECTO (NBE) Y DEL NIVEL DE EFECTO SEVERO (NES) DESCRITOS EN LAS NORMAS PARA LA PROTECCIÓN Y EL MANEJO DE SEDIMENTOS ACUÁTICOS DE ONTARIO, CANADÁ

\begin{tabular}{|c|c|c|c|c|c|c|c|c|c|c|c|c|c|c|}
\hline \multirow{2}{*}{$\begin{array}{l}\text { Elemento } \\
(\mathrm{mg} / \mathrm{kg})\end{array}$} & \multicolumn{8}{|c|}{ Este estudio } & \multicolumn{3}{|c|}{$\begin{array}{l}\text { Tarras-Wahlberg } \\
\text { et al. } 2001\end{array}$} & \multirow{2}{*}{$\begin{array}{c}\text { García et al. } \\
2012 \\
\text { Rango }\end{array}$} & \multicolumn{2}{|c|}{$\begin{array}{l}\text { Normas de } \\
\text { Ontario }\end{array}$} \\
\hline & $\mathrm{Am}$ & $\mathrm{A} 1$ & $\mathrm{C} 1$ & $\mathrm{C} 2$ & $\mathrm{~A} 2$ & A3 & $\mathrm{P} 1$ & $\mathrm{P} 2$ & $\mathrm{C} 2$ & $\mathrm{~A} 2$ & $\mathrm{P} 1$ & & NBE & NES \\
\hline $\mathrm{Mn}$ & 374 & 1362 & 1130 & 1539 & 1977 & 1488 & 1706 & 2079 & - & - & - & 809-1490 & 460 & 1100 \\
\hline $\mathrm{Zn}$ & 89.3 & 370 & 1040 & 3398 & 3716 & 8225 & 3595 & 3836 & 5100 & 3800 & 2400 & - & 120 & 820 \\
\hline $\mathrm{Pb}$ & 19.1 & 101 & 172 & 1745 & 693 & 3272 & 1174 & 1312 & 3200 & 2400 & 1400 & $440-1090$ & 31 & 250 \\
\hline $\mathrm{Cu}$ & 22.3 & 83.8 & 522 & 1683 & 622 & 2894 & 1390 & 1807 & 6200 & 1500 & 2100 & - & 16 & 110 \\
\hline $\mathrm{Ni}$ & 11.8 & 11.1 & 8.10 & 18.6 & 10.8 & 32.7 & 13.9 & 19.1 & - & - & - & - & 16 & 75 \\
\hline Co & 5.8 & 20.0 & 17.7 & 30.6 & 18.3 & 73.4 & 19.2 & 22.1 & - & - & - & - & - & - \\
\hline $\mathrm{Hg}$ & 0.03 & 0.11 & 0.26 & 2.32 & 0.23 & 2.64 & 2.25 & 2.96 & 0.85 & 0.22 & 0.66 & $0.45-0.82$ & 0.2 & 2 \\
\hline $\mathrm{Fe}(\%)$ & 2.17 & 4.47 & 4.31 & 6.47 & 3.97 & 13.06 & 4.79 & 5.64 & - & - & - & - & 2 & 4 \\
\hline $\mathrm{Al}(\%)$ & 1.68 & 3.56 & 2.80 & 1.12 & 2.32 & 0.78 & 1.81 & 2.13 & - & - & - & - & - & - \\
\hline
\end{tabular}


del $\mathrm{Hg}$ natural que se encuentra contenido en los suelos de la región, lo que incrementaria el flujo de $\mathrm{Hg}$ hacia las zonas bajas de las cuencas hidrográficas. Por consiguiente, tanto el incremento en la actividad minera artesanal como la deforestación pudieran ser responsables del aumento de $\mathrm{Hg}$ en los sedimentos de la sección media y baja del río Puyango.

El cuadro III también indica que los elementos $\mathrm{Mn}, \mathrm{Cu}, \mathrm{Pb}, \mathrm{Zn}, \mathrm{Hg}$ y Fe en los sedimentos de los sectores A2, C2, A3, P1 y P2 presentan concentraciones muy superiores (con excepción del $\mathrm{Hg}$ en A2) al nivel de bajo efecto (NBE) y al nivel de efecto severo (NES), reportados en las normas para la protección y el manejo de sedimentos acuáticos del Ministerio del Ambiente de Ontario (Persaud et al. 1993). Según esta norma, el NES indica que a estos niveles de concentración el sedimento es considerado altamente contaminado y puede tener un efecto adverso para los organismos bentónicos que habitan en ellos.

Aunque los sedimentos de los ríos Calera, Amarillo y Puyango muestran concentraciones de $\mathrm{Mn}, \mathrm{Cu}$, $\mathrm{Co}$ y Ni inferiores a las encontradas en sedimentos de zonas marino-costeras ubicadas en la Península de Baja California (México), también impactadas por la explotación minera del Cobre (Shumilin et al. 2011, Shumilin et al. 2013), las concentraciones de $\mathrm{Pb}$ y Zn en los sedimentos del Puyango son superiores a las de los sedimentos de esas zonas de México. Los resultados obtenidos en este estudio también indican que las concentraciones de $\mathrm{Mn}, \mathrm{Pb}, \mathrm{Cu}$ y $\mathrm{Zn}$ en los sedimentos del Puyango son mayores a las concentraciones de estos mismos elementos reportadas por Adánez et al. (2014) en los sedimentos de los ríos Odiel y Tinto (Mn 717 y 187 mg/kg, Pb 212 y 977 mg/kg, $\mathrm{Cu} 1220$ y $535 \mathrm{mg} / \mathrm{kg}$ y Zn 452 y $232 \mathrm{mg} / \mathrm{kg}$ ), ríos que drenan cuencas localizadas en la Faja Pirítica Ibérica (Huelva, España) y que han sido fuertemente afectados por las actividades mineras y el drenaje ácido de minas por más de 5000 años (Cánovas et al. 2007). De manera similar, las concentraciones de $\mathrm{Hg}$ total en los sedimentos después de las plantas de beneficio presentan valores muy parecidos a los encontrados en los sedimentos de ciertos ríos que han sido impactados por la minería aurífera artesanal en Ghana (valores entre 1.2 y $8.5 \mathrm{mg} / \mathrm{kg}$ ), en el sureste de Senegal (valor máximo de $9.9 \mathrm{mg} / \mathrm{kg}$ ) y en la región de La Mojana (valores entre 0.19 y $1,19 \mathrm{mg} / \mathrm{kg}$ ), al norte de Colombia (Niane et al. 2014, AdjeiKyereme et al. 2015, Pinedo-Hernández et al. 2015). Aunque el $\mathrm{Hg}$ que se reporta en este estudio es $\mathrm{Hg}$ total, es importante comentar que las cantidades de metil mercurio son mínimas en la sección alta y media de la cuenca del Puyango, debido a la toxicidad que representa el cianuro para las bacterias metiladoras de $\mathrm{Hg}$. Estudios realizados por Guimaraes et al. (2011) indican que el cianuro presente en las aguas del río Puyango reduce la actividad de las bacterias metiladoras y por ende la metilación de $\mathrm{Hg}$.

Debido a que este estudio sólo se realizó durante la época de sequía, los valores reportados pueden ser considerados como altos, dado que durante la época de alto caudal los sedimentos superficiales ricos en metales pesados suelen resuspenderse y fluir hacia los sectores más bajos de la cuenca (río Tumbes). De manera similar, el río Puyango es muy susceptible a los cambios en las precipitaciones producidas por el evento climático El Niño. Es decir, los impactos ambientales producidos por las plantas de beneficio pueden ser aún más severos en los años de extrema sequía (La Niña), debido a la ineficiencia que posee el río para transportar los sedimentos contaminados aguas abajo durante los periodos de bajas precipitaciones (Tarras-Wahlberg y Lane 2003).

\section{Relaciones lineales con el aluminio}

La figura 3 muestra los diagramas de dispersión en donde se presentan las concentraciones de los metales pesados determinados $v s$. las concentraciones de Al en cada una de las muestras de sedimento. Con el fin de visualizar el enriquecimiento de elementos en las zonas impactadas por la minería, se realizaron dos diagramas de dispersión para cada elemento en función de las concentraciones de Al. El primer diagrama (círculos negros), muestra las concentraciones de los metales determinados en los sedimentos de los sectores no impactados (Am y A1). Se excluyó al sector $\mathrm{C} 1$ de este análisis debido a que presentó evidencia de desarrollo de minería artesanal, a pesar de que está localizado en el río Calera aguas arriba de las plantas de beneficio, en la zona de alta montaña. En estos diagramas, las líneas discontinuas representan un intervalo de confianza de $95 \%$ para todos los datos de concentración colectados. Cuando los valores de concentración de los metales en los sedimentos se encuentran por arriba de estas líneas, existe un enriquecimiento antrópico del elemento en los sedimentos de ese sector en particular (Marmolejo-Rodríguez et al. 2007). El segundo diagrama (cuadrados blancos), muestra la relación existente entre los metales determinados y las concentraciones de $\mathrm{Al}$ en los sectores impactados por la actividad minera.

Como se observa en la figura 3, la relación entre las concentraciones de los elementos determinados y las concentraciones de Al en los sectores Am y A1 varía de altamente significativa $(\mathrm{p}<0.001)$ para el 

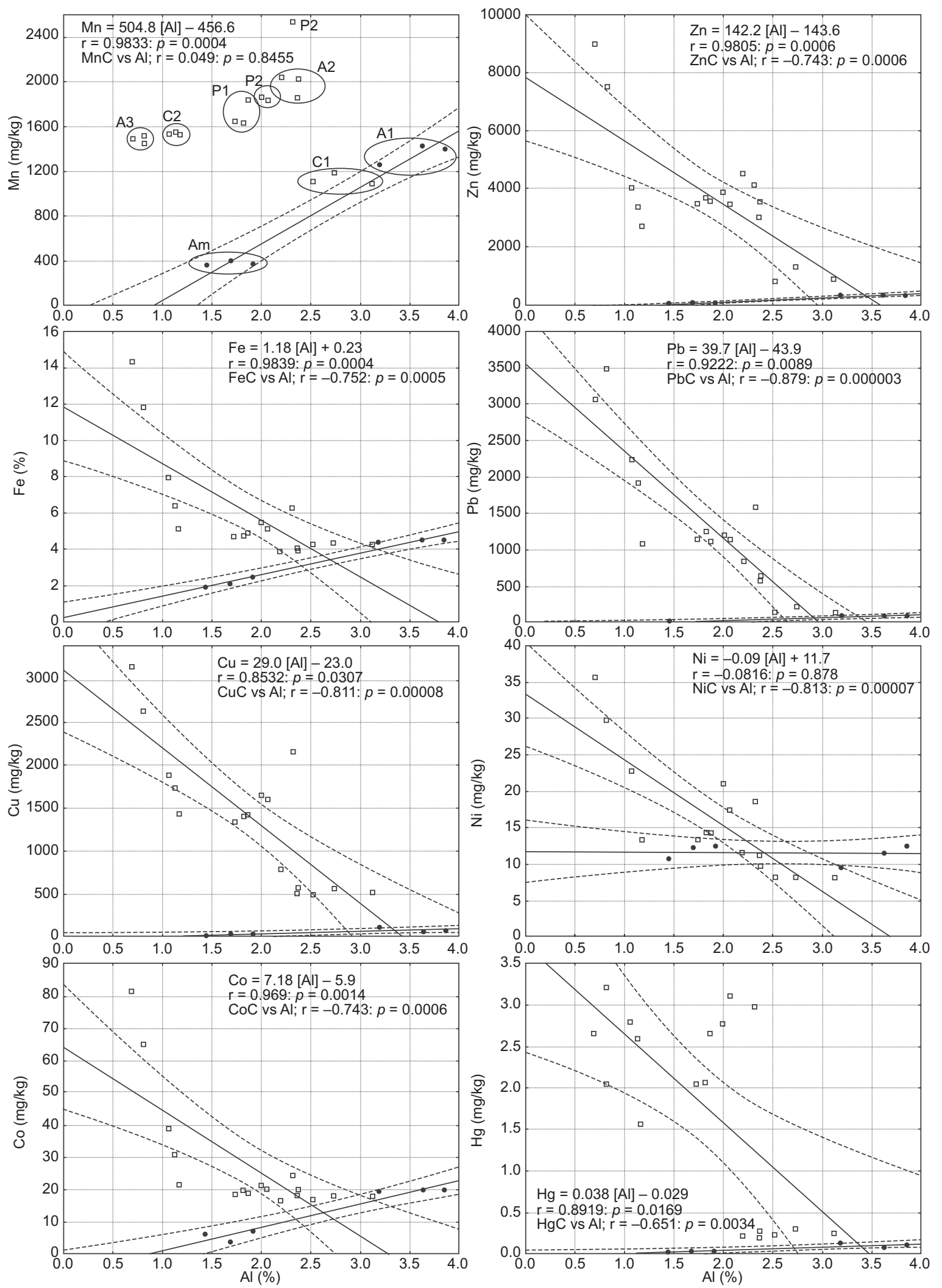

Fig. 3. Diagramas de dispersión de las concentraciones de los metales pesados determinados $v s$. las concentraciones de Aluminio tanto en sectores no impactados (círculos negros) como en sectores impactados (cuadros). MetalC denota la relación lineal en los sectores impactados por la minería 
Mn, Zn y Fe, muy significativa $(\mathrm{p}<0.01)$ para el $\mathrm{Pb}$ y el Co y significativa $(p<0.05)$ para el $\mathrm{Cu}$ y el $\mathrm{Hg}$. Asimismo, esta figura muestra que los sedimentos de las zonas impactadas presentan un enriquecimiento en los elementos $\mathrm{Mn}, \mathrm{Zn}, \mathrm{Fe}, \mathrm{Cu}, \mathrm{Pb}, \mathrm{Co}$ y $\mathrm{Hg}$, debido a que las concentraciones encontradas son superiores a los niveles que debería tener ese sedimento de acuerdo con su concentración de Al. De manera similar esta figura muestra que existe una relación negativa significativa $(\mathrm{p}<0.001)$ entre las concentraciones de $\mathrm{Zn}, \mathrm{Fe}, \mathrm{Cu}, \mathrm{Pb}$, Ni y $\mathrm{Co}$ y las concentraciones de $\mathrm{Al}$ en los sedimentos de los sectores impactados por la minería. Estudios realizados por Betancourt et al. (2005) demuestran que la cantidad de sólidos suspendidos que transportan los ríos Calera y Amarillo antes de las plantas de beneficio es muy baja $(3 \mathrm{mg} / \mathrm{L})$. Sin embargo, después de las plantas de beneficio, las concentraciones de material en suspensión se incrementan considerablemente en ambos ríos (por arriba de los $700 \mathrm{mg} / \mathrm{L}$ ). El material suspendido aportado por las plantas de beneficio (el cual sedimenta aguas abajo) es rico en metales pesados. Lo anterior debido a que dicho material es el producto de la molienda del mineral polimetálico en el que se encuentra contenido el oro. Por consiguiente, la disminución de las concentraciones de $\mathrm{Zn}, \mathrm{Fe}, \mathrm{Cu}, \mathrm{Pb}$, Ni y $\mathrm{Co}$ a medida de que se incrementan las concentraciones de Al en los sedimentos se debe a que los sólidos provenientes de las procesadoras de mineral se mezclan con sedimentos no contaminados ricos en $\mathrm{Al}$ (arci1las), los cuales son aportados por los tributarios de las secciones alta (Pindo y Ambocas) y media de la cuenca. Este hecho se evidencia claramente en los sedimentos colectados en el sector A3, los cuales poseen muy poco contenido de $\mathrm{Al}$ (entre $0.70 \mathrm{y}$ $0.82 \%$ ) y elevadas concentraciones de $\mathrm{Zn}, \mathrm{Fe}, \mathrm{Cu}$, $\mathrm{Pb}$, Ni y $\mathrm{Co}$, lo que indica que están constituidos principalmente por el mineral molido aportado por las plantas de beneficio.

Las concentraciones de Ni no mostraron una relación significativa con las concentraciones de $\mathrm{Al}$ en las muestras de sedimentos de los sectores no impactados (Fig. 3). Esto posiblemente se debe a que el ion $\mathrm{Ni}^{2+}$ es fácilmente desplazado por otros elementos de los sitios de intercambio que poseen los minerales arcillosos (Imhoff y Koppe 1981). Las concentraciones de Mn no mostraron una relación negativa con el $\mathrm{Al}$ en los sedimentos de los sectores impactados, posiblemente debido a que las cantidades de $\mathrm{Mn}$ contenidas en el mineral sulfuroso molido, que es aportado por las plantas de beneficio, son ligeramente superiores a las concentraciones basales de Mn que poseen los ríos no impactados de la zona de estudio. Las concentraciones de Mn en los sedimentos de los sectores impactados son sólo cuatro veces superiores a los valores base, mientras que las concentraciones de $\mathrm{Zn}, \mathrm{Cu}$ y $\mathrm{Pb}$ en estos mismos sedimentos fueron entre uno y dos órdenes de magnitud superiores a los valores base.

\section{Factores de enriquecimiento normalizados (FEN)}

Debido a que la composición litológica de la cuenca del río Puyango comprende depósitos masivos de sulfuros polimetálicos, los sedimentos colectados en los sectores no impactados por la minería poseen mayores concentraciones de metales pesados que las presentadas por otros sedimentos no impactados a nivel global, tales como los sedimentos de fondo del río Orinoco y sus tributarios (Mora et al. 2013). Por consiguiente, los niveles base de los elementos en los sedimentos impactados de esta cuenca deben ser calculados a partir de las ecuaciones obtenidas en los diagramas de dispersión realizados en los sectores no impactados (Am y A1), los cuales se muestran en la figura 3. Los factores de enriquecimiento normalizados (FEN), fueron calculados siguiendo el método utilizado por Marmolejo-Rodríguez et al. (2007), con la ecuación 1:

$\mathrm{FEN}=[\mathrm{Me}]_{\mathrm{S}} /[\mathrm{Me}]_{\mathrm{NB}}$

donde $[\mathrm{Me}]_{\mathrm{S}}$ representa la concentración del metal en una muestra dada y $[\mathrm{Me}]_{\mathrm{NB}}$ representa la concentración del metal que tendría una muestra no contaminada de acuerdo con su concentración de Al. Los valores de $[\mathrm{Me}]_{\mathrm{NB}}$ se obtuvieron a partir de las relaciones lineales obtenidas en los diagramas de dispersión realizados para cada elemento (metal vs. aluminio) en los sectores no impactados (Am y A1) y que se muestran en la ecuación 2:

$[\mathrm{Me}]_{\mathrm{NB}}=\mathrm{a}[\mathrm{Al}]+\mathrm{b}$

Debido a que no se encontró una relación lineal significativa entre las concentraciones de $\mathrm{Ni}$ y las de Al en los sedimentos de los sectores no impactados $(\mathrm{p}=0.8779)$, se tomó el valor promedio de Ni de los sedimentos colectados en los sectores Am y A1 (11.47 $\mathrm{mg} / \mathrm{kg}$ ) como el nivel base para el Ni. Para calcular los niveles base elementales para los sedimentos de los sectores $\mathrm{C} 2$ y A3, se interpoló la recta en el valor "0" de la ordenada $(b=0)$, dado que la ecuación resultante indicaba valores base negativos para casi todos los elementos estudiados en los sedimentos de dichos sectores. Esto se debe principalmente a 
que los sedimentos de ambos sectores se encuentran constituidos principalmente por mineral polimetálico molido, el cual posee una concentración de $\mathrm{Al}$ muy inferior a la que poseen los sedimentos de río.

La interpretación de los FEN fue llevada a cabo de acuerdo con la clasificación propuesta por Hakanson (1980), la cual ha sido también recientemente utilizada por Cobelo-García y Prego (2004) y por Marmolejo-Rodríguez et al. (2007). Esta clasificación expone que para valores de $\mathrm{FEN}<2$, la contaminación es baja o despreciable, para valores de FEN entre 2 y 3 la contaminación es moderada, mientras que valores de FEN $>3$ indican la existencia de una severa contaminación con el metal en el sedimento estudiado. El cuadro IV muestra un FEN cercano a 1 para todos los elementos determinados en los sedimentos de los sectores Am y A1, lo que indica que el contenido de metales pesados en estos sedimentos se debe a fuentes geológicas naturales. Sin embargo, los sedimentos colectados en el sector del río Calera antes de las plantas de beneficio (sector $\mathrm{C} 1$ ) presentan una contaminación que va de moderada a severa (valores de FEN entre 2 y 9) en los elementos $\mathrm{Zn}, \mathrm{Cu}, \mathrm{Pb}$ y $\mathrm{Hg}$, lo cual señala que existe un impacto provocado por la pequeña minería en esta zona de alta montaña. Los sedimentos colectados en los ríos Calera y Amarillo después de las plantas de beneficio (sectores $\mathrm{C} 2$ y A2) presentaron factores de enriquecimiento altos ( $\geq 10$ ) para los metales $\mathrm{Zn}, \mathrm{Cu}, \mathrm{Pb}$ y Hg, lo que indica que estos sedimentos se encuentran severamente contaminados por dichos metales pesados. Los sedimentos colectados en el sector A3 presentaron altos factores de enriquecimiento para todos los elementos estudiados. Inclusive, estos factores de enriquecimiento fueron los más altos para toda la cuenca del Puyango (FEN mínimo de 2.85 para el Ni y máximo de 182 para el $\mathrm{Cu}$ ). Lo anterior indica que la mayor parte del sedimento en este sector se encuentra constituido por mineral polimetálico proveniente de las plantas de beneficio, el cual posee altas cantidades de $\mathrm{Hg}$ como consecuencia del proceso de amalgamación del oro.

Hacia la sección media y baja de la cuenca del Puyango (sectores P1 y P2), no existe una alta variabilidad en los FEN determinados en los sedimentos. En estas secciones, la contaminación por Ni resultó ser despreciable $(\mathrm{FEN} \leq 2)$, mientras que el Fe presentó un ligero enriquecimiento. Los elementos más enriquecidos en estos sedimentos (FEN $\geq 10)$ fueron el $\mathrm{Hg}$, el $\mathrm{Cu}$, el $\mathrm{Pb}$ y el $\mathrm{Zn}$, respectivamente. Incluso, los FEN determinados para el $\mathrm{Pb}$ fueron superiores a los encontrados en sedimentos marino-costeros (valor mínimo de 12.5 y máximo de 22.4) de la región minera de Santa Rosalía (Baja California, México), en la cual se ha desarrollado una intensa actividad minera e industrial de $\mathrm{Cu}$ (Shumilin et al. 2013). Los sedimentos de la sección baja de la cuenca resultaron estar también severamente contaminados con $\mathrm{Mn}$ (FEN de 3.35). Estudios realizados por Betancourt et al. (2015) han demostrado que existe un decrecimiento de la inteligencia en niños que habitan la sección baja de la cuenca, principalmente por la ingesta del agua del río Puyango, que posee cantidades considerables de $\mathrm{Mn}$ en la fase particulada. Dado que en los sectores bajos de la cuenca (sector Gramadal-Las Vegas), el clima es semiárido, las fuentes de agua son muy limitadas, lo que promueve el consumo de agua del río Puyango por parte de las comunidades rurales que habitan en esa zona (Betancourt et al. 2005). Dado que los sedimentos severamente contaminados con metales pesados son resuspendidos durante la época de alto caudal del río, el consumo de agua del río Puyango sin ningún tipo de tratamiento, incrementa considerablemente el riesgo de intoxicación por metales pesados en las poblaciones que utilizan esta fuente de agua para su sustento.

CUADRO IV. PROMEDIO DE LOS FACTORES DE ENRIQUECIMIENTO NORMALIZADOS CALCULADOS PARA LOS SEDIMENTOS COLECTADOS EN LOS SECTORES DE ESTUDIO

\begin{tabular}{lrrrrrrrr}
\hline Sector & $\mathrm{Mn}$ & $\mathrm{Zn}$ & $\mathrm{Fe}$ & $\mathrm{Pb}$ & $\mathrm{Cu}$ & $\mathrm{Ni}$ & $\mathrm{Co}$ & $\mathrm{Hg}$ \\
\hline $\mathrm{Am}$ & 1.01 & 0.99 & 0.98 & 1.09 & 0.87 & 1.03 & 0.98 & 0.93 \\
$\mathrm{C} 1$ & 1.20 & 4.16 & 1.23 & 2.63 & 9.12 & 0.71 & 1.27 & 3.44 \\
$\mathrm{C} 2$ & 3.89 & 32.32 & 4.18 & 62.89 & 70.55 & 1.62 & 5.28 & 73.75 \\
$\mathrm{~A} 1$ & 1.02 & 1.03 & 1.01 & 1.06 & 1.07 & 0.97 & 1.03 & 1.05 \\
$\mathrm{~A} 2$ & 2.79 & 20.29 & 1.34 & 14.70 & 14.27 & 0.94 & 1.71 & 3.87 \\
$\mathrm{~A} 3$ & 5.47 & 117.07 & 11.75 & 173.73 & 182.03 & 2.85 & 18.93 & 120.97 \\
P1 & 3.73 & 31.66 & 2.02 & 42.39 & 47.16 & 1.21 & 2.71 & 56.55 \\
P2 & 3.35 & 24.28 & 2.05 & 32.32 & 46.42 & 1.66 & 2.36 & 57.44 \\
\hline
\end{tabular}




\section{CONCLUSIONES}

Los sedimentos de la sección media y baja del río Puyango se encuentran severamente contaminados (FEN $\geq 3$ ) con los elementos $\mathrm{Hg}, \mathrm{Cu}, \mathrm{Pb}, \mathrm{Mn}$ y Zn como consecuencia de la descargas de lixiviados mineros hacia los principales tributarios del Puyango (ríos Calera y Amarillo). Estos lixiviados poseen una alta concentración de $\mathrm{Hg}$ y otros metales pesados, que son el resultado del proceso de molienda del mineral sulfuroso polimetálico procedente de las minas. Las relaciones negativas encontradas entre las concentraciones de metales pesados y las concentraciones de $\mathrm{Al}$ en los sedimentos de los sitios impactados, revelan que la mayor parte del sedimento en estos sitios se encuentra constituido por mineral polimetálico. Los sedimentos colectados en la sección baja de la cuenca no mostraron evidencia de enriquecimiento en Ni. Sin embargo, estos sedimentos también presentan una moderada contaminación con Co y Fe, los cuales son también incorporados por los lixiviados provenientes de las plantas de beneficio del mineral aurífero. Las altas concentraciones de $\mathrm{Hg}, \mathrm{Cu}, \mathrm{Pb}, \mathrm{Zn}, \mathrm{Mn}$, Co y $\mathrm{Fe}$ encontradas en estos sedimentos pueden tener un efecto adverso para la biota y para la salud de las poblaciones humanas que residen en la sección baja de la cuenca. Lo anterior, debido a que el agua del río Puyango es utilizada para consumo como consecuencia de la poca disponibilidad de este recurso en la región.

\section{AGRADECIMIENTOS}

Este trabajo fue totalmente financiado por la Secretaría de Educación Superior, Ciencia, Tecnología e Innovación de la República del Ecuador y por la Universidad Nacional de Loja.

\section{REFERENCIAS}

Adánez P., Llamas J.F., Locutura J. y García A. (2014). Estudio geoquímico de los sedimentos de llanura de inundación en la cuenca de los ríos Tinto y Odiel (Huelva). Bol. Geol. Min. 125, 585-599.

Adjei-Kyereme Y., Donkor A.K., Golow A.A., Yeboah P.O. y Pwamang J. (2015). Mercury concentrations in water and sediments in rivers impacted by artisanal gold mining in the Asutifi district, Ghana. Res. J. Chem. Environ. Sci. 3, 40-48.

APHA (2012). Standard methods for the examination of water and wastewater. 22a ed. American Public Health Association, Washington, EUA, 1360 pp.
ARCOM (2016). Plan nacional de desarrollo del sector minero. Agencia de Regulación y Control Minero [en línea]. https://drive.google.com/ file/d/0B9t02UvtK83 SRTJJekJsdk1 CeTg/view $18 / 10 / 2016$

Betancourt O., Narváez A. y Roulet M. (2005). Smallscale gold mining in the Puyango River basin, southern Ecuador: A study of environmental impacts and human exposures. EcoHealth 2, 323-332.

DOI: $10.1007 / \mathrm{s} 10393-005-8462-4$

Betancourt O., Tapia M. y Méndez I. (2015). Decline of general intelligence in children exposed to Manganese from mining contamination in Puyango River basin, southern Ecuador. EcoHealth 12, 453-460.

DOI: $10.1007 / \mathrm{s} 10393-015-1027-2$

Cánovas C.R., Olías M., Nieto J.M., Sarmiento A.M. y Cerón J.C. (2007). Hydrogeochemical characteristics of the Tinto and Odiel Rivers (SW Spain). Factors controlling metal contents. Sci. Total Environ. 373, 363-382.

DOI: $10.1016 /$ j.scitotenv.2006.11.022

Cobelo-García A. y Prego R. (2004). Influence of point sources on trace metal contamination and distribution in a semi-enclosed industrial embayment: The Ferrol Ria (NW Spain). Estuar. Coast. Shelf Sci. 60, 695-703. DOI: 10.1016/j.ecss.2004.03.008

EPA (1996). Acid digestion of sediments, sludges and soils. Method 3050B. Environmental Protection Agency [en línea]. https://www.epa.gov/sites/production/ files/2015-06/documents/epa-3050b.pdf 14/01/2016

García M.E., Betancourt O., Cueva E. y Gimaraes J.R.D. (2012). Mining and seasonal variation of the metals concentration in the Puyango River basin-Ecuador. J. Environ. Protect. 3, 1542-1550.

DOI: $10.4236 /$ jep. 2012.311170

Gobierno Provincial Autónomo de El Oro (2014). Plan de desarrollo y ordenamiento territorial de la Provincia de El Oro, 2014-2025, 479 pp [en línea]. http://www.eloro.gob.ec/wp-content/uploads/2013/12/PDOT_2014. pdf 14/01/2016

Guimaraes J.R.D., Betancourt O., Rodrigues M., Barriga R., Cueva E. y Betancourt S. (2011). Long-range effect of cyanide on mercury methylation in a gold mining area in southern Ecuador. Sci. Total Environ. 409, 5026-5033.

DOI: $10.1016 /$ j.scitotenv.2011.08.021

Hakanson L. (1980). An ecological risk index for aquatic pollution control. A sedimentological approach. Water Res. 14, 975-1001.

DOI: $10.1016 / 0043-1354(80) 90143-8$

Imhoff K.R. y Koppe P. (1981). The fate of heavy metals in the Ruhr system and their influence on drinking water quality. Water Sci. Technol. 13, 211-225. 
Kaasalainen M. y Yli-Halla M. (2003). Use of sequential extraction to assess metal partitioning in soils. Environ. Pollut. 126, 225-233.

DOI: 10.1016/S0269-7491(03)00191-X

Marmolejo-Rodríguez A., Prego R., Meyer-Willerer A., Shumilin E. y Cobelo-García A. (2007). Total and labile metals in surface sediments of the tropical river-estuary system of Marabasco (Pacific coast of Mexico): Influence of an iron mine. Mar. Pollut. Bull. $55,459-468$.

DOI: 10.1016/j.marpolbul.2007.09.008

Mainville N., Webb J., Lucotte M., Davidson R., Betancourt O., Cueva E. y Mergler D. (2006). Decrease of soil fertility and release of mercury following deforestation in the Andean Amazon, Napo River Valley, Ecuador. Sci. Total Environ. 368, 88-98.

DOI: 10.1016/j.scitotenv.2005.09.064

Mora A., Alfonso J.A., Baquero J.C., Handt H. y Vásquez Y. (2013) Elementos mayoritarios, minoritarios y traza en muestras de sedimentos del medio y bajo río Orinoco, Venezuela. Rev. Int. Contam. Ambie. 29, 165-178.

Niane B., Moritz R., Guédron S., Ngom P.M., Pfeifer H.R., Mall I. y Poté J. (2014). Effect of recent artisanal small-scale gold mining on the contamination of surface river sediment: Case of Gambia River, Kedougou region, southeastern Senegal. J. Geochem. Explor. 144, 517-527.

DOI: $10.1016 /$ j.gexplo.2014.03.028

Persaud D., Jaagumagi R. y Hayton A. (1993). Guidelines for the protection and management of aquatic sediment quality in Ontario. Ontario Ministry of the Environment. Ottawa, Canadá, 23 pp.

Pinedo-Hernández J., Marrugo-Negrete J. y Díez S. (2015). Speciation and bioavailability of mercury in sediments impacted by gold mining in Colombia. Chemosphere 119, 1289-1295.

DOI: 10.1016/j.chemosphere.2014.09.044
Shumilin E., Gordeev V., Rodríguez G., Demina L. y Choumiline K. (2011). Assessment of geochemical mobility of metals in surface sediments of the Santa Rosalia mining region, western gulf of California. Arch. Environ. Contam. Toxicol. 60, 8-25.

DOI: $10.1007 / \mathrm{s} 00244-010-9532-3$

Shumilin E., Jiménez A.R. y López-López S. (2013). Anthropogenic contamination of metals in sediments of the Santa Rosalia Harbor, Baja California peninsula. Bull. Environ. Contam. Toxicol. 90, 333-337.

DOI: $10.1007 / \mathrm{s} 00128-012-0923-1$

Tarras-Wahlberg N.H. (2002). Environmental management of small-scale and artisanal mining: The PortoveloZaruma goldmining area, southern Ecuador. J. Environ. Manage. 65, 165-179.

DOI: $10.1006 /$ jema.2002.0542

Tarras-Wahlberg N.H. y Lane S.N. (2003). Suspended sediment yield and metal contamination in a river catchment affected by El Niño events and gold mining activities: The Puyango River basin, southern Ecuador. Hydrol. Process. 17, 3101-3123.

DOI: 10.1002/hyp.1297

Tarras-Wahlberg N.H., Flachier A., Fredriksson G., Lane S., Lundberg B. y Sangfors O. (2000). Environmental impact of small-scale and artisanal gold mining in southern Ecuador: Implications for the setting of environmental standards and for the management of small-scale mining operations. Ambio 29, 484-491. DOI: 10.1579/0044-7447-29.8.484

Tarras-Wahlberg N.H., Flachier A., Lane S.N. y Sangfors O. (2001). Environmental impacts and metal exposure of aquatic ecosystems in rivers contaminated by small scale gold mining: the Puyango River basin, southern Ecuador. Sci. Total Environ. 278, 239-261. DOI: 10.1016/S0048-9697(01)00655-6 